\title{
ROBUST ADAPTIVE BEAMFORMING USING PROBABILITY-CONSTRAINED OPTIMIZATION
}

\author{
Sergiy A. Vorobyov \\ Yue Rong \\ Alex B. Gershman
Communication Systems Group, Darmstadt University of Technology
Merckstr. 25, 64283 Darmstadt, Germany

\begin{abstract}
Recently, robust minimum variance (MV) beamforming which optimizes the worst-case performance has been proposed in [1], [2]. The worst-case approach, however, might be overly conservative in practical applications. In this paper, we propose a more flexible approach that formulates the robust adaptive beamforming problem as a probability-constrained optimization problem with homogeneous quadratic cost function. Unlike the general probability-constrained problem which can be nonconvex and NP-hard, our problem can be reformulated as a convex nonlinear programming (NLP) problem, and efficiently solved using interiorpoint methods. Simulation results show an improved robustness of the proposed beamformer as compared to the existing state-of-the-art robust adaptive beamforming techniques.
\end{abstract}

\section{INTRODUCTION}

In practical applications of adaptive beamforming, the robustness against a mismatch between the presumed and actual signal steering vectors is one of the most critical issues [1]-[4]. Robust adaptive MV beamforming algorithms proposed in [1] and [2] explicitly model the unknown mismatch between the presumed and actual steering vectors and obtain the beamformer weight vector by optimizing the performance for the worst-case mismatch. However, this approach may be overly conservative in practical applications, especially taking into account that the worst-case mismatch may actually occur very seldom.

In this paper, we use a less conservative robust approach, which guarantees the robustness against the signal steering vector mismatch with a certain selected probability. The proposed approach is quite universal and can be applied to other related problems as well [5]-[6]. It is based on the probability-constrained optimization that is also sometimes called chance programming [7]. The corresponding probability-constrained optimization problem is convex under the assumption that the steering vector mismatch is Gaussian, and it can be solved by applying nonlinear programming
(NLP) techniques which use the well established interiorpoint methods. Simulation results compare the performance of the proposed beamformer to that of the worst-case based beamformer of [1] and several other popular adaptive beamformers under the standard choice of the robustness parameters.

\section{PROBLEM FORMULATION}

The output of a narrowband beamformer is given by

$$
y(k)=\boldsymbol{w}^{H} \boldsymbol{x}(k)
$$

where $k$ is the sample index, $\boldsymbol{x}(k)=\left[x_{1}(k), \ldots, x_{M}(k)\right]^{T}$ is the complex vector of array observations, $\boldsymbol{w}=\left[w_{1}, \ldots\right.$, $\left.w_{M}\right]^{T}$ is the complex vector of beamformer weights, $M$ is the number of array sensors, and $(\cdot)^{T}$ and $(\cdot)^{H}$ denote the transpose and Hermitian transpose, respectively. The observation vector is given by

$$
\boldsymbol{x}(k)=s(k) \boldsymbol{a}+\boldsymbol{i}(k)+\boldsymbol{n}(k)
$$

where $s(k)$ is the desired signal waveform, $\boldsymbol{a}$ is the signal steering vector, and $\boldsymbol{i}(k)$ and $\boldsymbol{n}(k)$ are the interference and noise components, respectively. The optimal weight vector can be obtained by means of maximization of the signal-tointerference-plus-noise ratio (SINR)

$$
\mathrm{SINR}=\frac{\sigma_{\mathrm{s}}^{2}\left|\boldsymbol{w}^{H} \boldsymbol{a}\right|^{2}}{\boldsymbol{w}^{H} \boldsymbol{R}_{\mathrm{i}+\mathrm{n}} \boldsymbol{w}}
$$

where $\boldsymbol{R}_{\mathrm{i}+\mathrm{n}}$ is the $M \times M$ interference-plus-noise covariance matrix and $\sigma_{\mathrm{s}}^{2}$ is the signal variance. In practical applications, the exact knowledge of $\boldsymbol{R}_{\mathrm{i}+\mathrm{n}}$ is unavailable because of the presence of the signal component and/or finite observation time. Hence, the sample covariance matrix

$$
\hat{\boldsymbol{R}}=\frac{1}{K} \sum_{k=1}^{K} \boldsymbol{x}(k) \boldsymbol{x}(k)^{H}
$$

is usually used instead of $\boldsymbol{R}_{\mathrm{i}+\mathrm{n}}$, where $K$ is the training sample size. Then, the sample version of the problem of maximizing SINR can be written as

$$
\min _{\boldsymbol{w}} \boldsymbol{w}^{H} \hat{\boldsymbol{R}} \boldsymbol{w} \quad \text { subject to } \quad \boldsymbol{w}^{H} \boldsymbol{a}=1 .
$$


The solution of this problem is referred to as the sample matrix inversion (SMI) based minimum variance distortionless response (MVDR) beamformer whose weight vector is given by [8]

$$
\boldsymbol{w}_{\mathrm{SMI}}=\alpha \hat{\boldsymbol{R}}^{-1} \boldsymbol{a}
$$

where $\alpha=1 / \boldsymbol{a}^{H} \hat{\boldsymbol{R}}^{-1} \boldsymbol{a}$.

An essential shortcoming of the MVDR beamformer (5) is that it is not robust against a mismatch between the presumed and actual signal steering vectors $\boldsymbol{a}$ and $\tilde{\boldsymbol{a}}$, respectively. In [1] and [2], the actual (mismatched) steering vector $\tilde{\boldsymbol{a}}$ has been explicitly modelled as

$$
\tilde{\boldsymbol{a}}=\boldsymbol{a}+\boldsymbol{\delta} \neq \boldsymbol{a}
$$

where $\delta$ denotes an unknown complex vector which describes the effect of steering vector distortions (the so-called mismatch vector). In this case, equation (2) should be rewritten as [1]

$$
\mathrm{SINR}=\frac{\sigma_{\mathrm{s}}^{2}\left|\boldsymbol{w}^{H} \tilde{\boldsymbol{a}}\right|^{2}}{\boldsymbol{w}^{H} \boldsymbol{R}_{\mathrm{i}+\mathrm{n}} \boldsymbol{w}}
$$

and the problem (4) should also be reformulated taking into account the mismatch vector $\boldsymbol{\delta}$.

It has been assumed in [1] and [2] that $\delta$ is an unknown deterministic vector that is bounded in its norm by some known positive constant

$$
\|\boldsymbol{\delta}\| \leq \varepsilon
$$

where $\|\cdot\|$ denotes Euclidian norm of vector. Then, the actual signal steering vector belongs to the uncertainty set

$$
\mathcal{A}(\varepsilon) \triangleq\{\tilde{\boldsymbol{a}} \mid \tilde{\boldsymbol{a}}=\boldsymbol{a}+\boldsymbol{\delta}, \quad\|\boldsymbol{\delta}\| \leq \varepsilon\}
$$

and the design of robust adaptive beamforming boils down to solving the MVDR problem for the worst-case steering vector. It is easy to verify that such worst-case vector $\tilde{\boldsymbol{a}}$ lies on the boundary of the set $\mathcal{A}(\varepsilon)$ [1]. A beamformer similar to [1] and [2] has been derived in [9] from a covariance fitting (rather than the maximum SINR) perspective. Note that the worst-case approach studied in [1], [2], and [9] may be overly conservative, since the worst-case mismatch may actually occur quite seldom in practice.

\section{ROBUST ADAPTIVE BEAMFORMING VIA PROBABILITY-CONSTRAINED OPTIMIZATION}

In this work, we assume that the mismatch vector $\delta$ is an unknown random vector with known probability density function. Then, the robust formulation of adaptive beamformer based on the probability-constrained optimization can be written as

$$
\min _{\boldsymbol{w}} \boldsymbol{w}^{H} \hat{\boldsymbol{R}} \boldsymbol{w} \quad \text { subject to } \quad \operatorname{Pr}\left\{\left|\boldsymbol{w}^{H} \tilde{\boldsymbol{a}}\right| \geq 1\right\} \geq p
$$

where $p$ is a certain probability value which can be selected according to the quality of service (QoS) requirements, and $\operatorname{Pr}\{\cdot\}$ stands for the probability operator.

The problem (6) becomes mathematically tractable if we additionally assume a specific analytic form for the probability operator $\operatorname{Pr}\{\cdot\}$ and make some approximations. Hereafter, we assume that $\delta$ is drawn from a complex circularly symmetric Gaussian distribution with zero mean and covariance matrix $C_{\delta}$ [10], i.e.

$$
\boldsymbol{\delta} \sim \mathcal{C N}\left(\mathbf{0}_{M}, \boldsymbol{C}_{\boldsymbol{\delta}}\right)
$$

The covariance matrix $\boldsymbol{C}_{\boldsymbol{\delta}}$ captures the second-order statistics of the uncertainties in the steering vector. In some applications, $\boldsymbol{C}_{\boldsymbol{\delta}}$ can be assumed to be a diagonal matrix of the form $\sigma_{\delta}^{2} \boldsymbol{I}_{M}$, where $\sigma_{\delta}^{2}$ denotes the variance [10]. However, in a number of applications $C_{\delta}$ is not a diagonal matrix. For example, the wavefront can be distorted by its propagation through an inhomogeneous medium, and this may lead to independent-increment wavefront phase distortions [11]. In the latter case, the entries of mismatch vector become correlated and $\boldsymbol{C}_{\boldsymbol{\delta}}$ is not diagonal. Another example is signal propagation through a Ricean channel, where the covariance matrix of the mismatch vector may depend on the spatial distribution of the scatterers [12].

Using (7), it is easy to show that the random variable $\boldsymbol{w}^{H}(\boldsymbol{a}+\boldsymbol{\delta})$ has the following distribution

$$
\boldsymbol{w}^{H}(\boldsymbol{a}+\boldsymbol{\delta}) \sim \mathcal{C N}\left(\boldsymbol{w}^{H} \boldsymbol{a},\left\|\boldsymbol{C}_{\boldsymbol{\delta}}^{1 / 2} \boldsymbol{w}\right\|^{2}\right) .
$$

Hence, the random variable $\left|\boldsymbol{w}^{H}(\boldsymbol{a}+\boldsymbol{\delta})\right|$ has Ricean distribution. We will approximate the constraint in (6) by the following constraints

$$
\begin{aligned}
& \operatorname{Pr}\left\{\left|\operatorname{Re}\left\{\boldsymbol{w}^{H} \tilde{\boldsymbol{a}}\right\}\right| \geq \beta\right\} \geq p \\
& \operatorname{Pr}\left\{\left|\operatorname{Im}\left\{\boldsymbol{w}^{H} \tilde{\boldsymbol{a}}\right\}\right| \geq \beta\right\} \geq p
\end{aligned}
$$

where $\beta$ can be found by solving the equation

$$
1=\left|\boldsymbol{w}^{H} \tilde{\boldsymbol{a}}\right|^{2}=\operatorname{Re}\left\{\boldsymbol{w}^{H} \tilde{\boldsymbol{a}}\right\}^{2}+\operatorname{Im}\left\{\boldsymbol{w}^{H} \tilde{\boldsymbol{a}}\right\}^{2}=2 \beta^{2}
$$

that is, $\beta=1 / \sqrt{2}$.

Using the aforementioned approximation of the constraint in (6), the optimization problem (6) can be rewritten as

$$
\begin{array}{ll}
\min _{\boldsymbol{w}} \boldsymbol{w}^{H} \hat{\boldsymbol{R}} \boldsymbol{w} \\
\text { subject to } & \operatorname{Pr}\left\{\left|\operatorname{Re}\left\{\boldsymbol{w}^{H} \tilde{\boldsymbol{a}}\right\}\right| \geq 1 / \sqrt{2}\right\} \geq p \\
& \operatorname{Pr}\left\{\left|\operatorname{Im}\left\{\boldsymbol{w}^{H} \tilde{\boldsymbol{a}}\right\}\right| \geq 1 / \sqrt{2}\right\} \geq p
\end{array}
$$

where

$$
\begin{aligned}
& \operatorname{Re}\left\{\boldsymbol{w}^{H} \tilde{\boldsymbol{a}}\right\} \sim \mathcal{N}\left(\operatorname{Re}\left\{\boldsymbol{w}^{H} \boldsymbol{a}\right\},\left\|\boldsymbol{C}_{\boldsymbol{\delta}}^{1 / 2} \boldsymbol{w}\right\|^{2} / 2\right) \\
& \operatorname{Im}\left\{\boldsymbol{w}^{H} \tilde{\boldsymbol{a}}\right\} \sim \mathcal{N}\left(\operatorname{Im}\left\{\boldsymbol{w}^{H} \boldsymbol{a}\right\},\left\|\boldsymbol{C}_{\boldsymbol{\delta}}^{1 / 2} \boldsymbol{w}\right\|^{2} / 2\right) .
\end{aligned}
$$




\section{CONVEXITY}

Let us first establish the convexity of problem (9). Towards this end, the following lemma will be needed.

LEMMA 1: Let vectors $\boldsymbol{v}_{1}, \ldots, \boldsymbol{v}_{n}$ have a joint real Gaussian distribution and

$$
\mathrm{E}\left\{\left(\boldsymbol{v}_{i}-\mathrm{E}\left\{\boldsymbol{v}_{i}\right\}\right)\left(\boldsymbol{v}_{l}-\mathrm{E}\left\{\boldsymbol{v}_{l}\right\}\right)^{T}\right\}=r_{i l} \boldsymbol{B}, \quad \forall i, l
$$

where $r_{i l}$ are some constants; $i, l=1, \ldots, n$; and the matrix $\boldsymbol{B}$ describes a common covariance structure of the given vectors. Then the set

$\mathcal{K}(p)=\left\{\boldsymbol{x} \mid \operatorname{Pr}\left\{\boldsymbol{v}_{1}^{T} \boldsymbol{x} \geq \eta_{1} \wedge \ldots \wedge \boldsymbol{v}_{n}^{T} \boldsymbol{x} \geq \eta_{n}\right\} \geq p\right\}$

is convex for $p \geq 0.5$. Here $\mathrm{E}\{\cdot\}$ stands for expectation operator, $\wedge$ denotes the set intersection operation, $0<p \leq$ 1 , and $\eta_{i}$ are some constants.

Proof: See [7, p. 312]

Now we can prove the convexity of the optimization problem (9).

THEOREM 1: If (7) is valid and $p \in[0.5,1)$ then the optimization problem (9) is convex.

PROOF: The objective function of (9) is a quadratic form, where $\hat{\boldsymbol{R}}$ is a positive definite matrix. Thus, it is convex.

The probability constraints of (9) share the same structure. Then, it is enough to prove that one of them is convex. Let us rewrite the first constraint of (9) as

$$
\operatorname{Pr}\left\{\operatorname{Re}\left\{\boldsymbol{w}^{H} \tilde{\boldsymbol{a}}\right\} \geq 1 / \sqrt{2} \wedge-\operatorname{Re}\left\{\boldsymbol{w}^{H} \tilde{\boldsymbol{a}}\right\} \geq 1 / \sqrt{2}\right\} \geq p .
$$

To be consistent with the notations used in Lemma 1, let us denote

$$
\begin{aligned}
\boldsymbol{v} & :=\left[\operatorname{Re}\{\tilde{\boldsymbol{a}}\}^{T}, \operatorname{Im}\{\tilde{\boldsymbol{a}}\}^{T}\right]^{T} \\
\boldsymbol{x} & :=\left[\operatorname{Re}\{\boldsymbol{w}\}^{T}, \operatorname{Im}\{\boldsymbol{w}\}^{T}\right]^{T} \\
\eta & :=1 / \sqrt{2} .
\end{aligned}
$$

Then, the constraint (12) can be equivalently written as

$$
\operatorname{Pr}\left\{\boldsymbol{v}^{T} \boldsymbol{x} \geq \eta \wedge-\boldsymbol{v}^{T} \boldsymbol{x} \geq \eta\right\} \geq p .
$$

Since the vectors $\boldsymbol{v}$ and $-\boldsymbol{v}$ have joint Gaussian distribution with the common covariance matrix

$$
B:=-\frac{1}{2}\left[\begin{array}{cc}
C_{\delta} & 0 \\
0 & C_{\delta}
\end{array}\right]
$$

we can see that Lemma 1 can be applied. Thus, the convexity of the first constraint of (9) is proved if $p \in[0.5,1)$. The convexity of the second constraint can be proved similarly.

Summarizing, the objective function of the problem (9) is convex and the constraints are convex provided that $p \in$ $[0.5,1)$. This completes the proof of the theorem.

It follows from Theorem 1 that the problem (9) has only one global minimum if (7) is valid and $p \in[0.5,1)$. To find this minimum, we need to convert (9) into its deterministic equivalent form.

\section{IMPLEMENTATION}

We can rewrite the left hand side of the first probability constraint of the problem (9) as

$$
\begin{aligned}
\operatorname{Pr}\left\{\left|\operatorname{Re}\left\{\boldsymbol{w}^{H} \tilde{\boldsymbol{a}}\right\}\right| \geq 1 / \sqrt{2}\right\} \\
=1-\operatorname{Pr}\left\{\left|\operatorname{Re}\left\{\boldsymbol{w}^{H} \tilde{\boldsymbol{a}}\right\}\right| \leq 1 / \sqrt{2}\right\} .
\end{aligned}
$$

Using (10) and the standard error function for Gaussian distribution

$$
\operatorname{erf}(x)=\frac{2}{\sqrt{\pi}} \int_{0}^{x} e^{-t^{2}} d t
$$

we can further write

$$
\begin{aligned}
\operatorname{Pr}\left\{\left|\operatorname{Re}\left\{\boldsymbol{w}^{H} \tilde{\boldsymbol{a}}\right\}\right|\right. & \leq 1 / \sqrt{2}\}=\operatorname{Pr}\left\{\operatorname{Re}\left\{\boldsymbol{w}^{H} \tilde{\boldsymbol{a}}\right\} \leq 1 / \sqrt{2}\right\} \\
& -\operatorname{Pr}\left\{\operatorname{Re}\left\{\boldsymbol{w}^{H} \tilde{\boldsymbol{a}}\right\} \leq-1 / \sqrt{2}\right\} \\
& =\frac{1}{2}\left[\operatorname{erf}\left(\frac{\sqrt{1 / 2}-\operatorname{Re}\left\{\boldsymbol{w}^{H} \boldsymbol{a}\right\}}{\left\|\boldsymbol{C}_{\boldsymbol{\delta}}^{1 / 2} \boldsymbol{w}\right\|}\right)\right. \\
& \left.-\operatorname{erf}\left(\frac{-\sqrt{1 / 2}-\operatorname{Re}\left\{\boldsymbol{w}^{H} \boldsymbol{a}\right\}}{\left\|\boldsymbol{C}_{\boldsymbol{\delta}}^{1 / 2} \boldsymbol{w}\right\|}\right)\right]
\end{aligned}
$$

Using (13) and (14), the first constraint of the problem (9) can be written in the following equivalent deterministic form

$$
\begin{aligned}
& \operatorname{erf}\left(\frac{-\sqrt{1 / 2}-\operatorname{Re}\left\{\boldsymbol{w}^{H} \boldsymbol{a}\right\}}{\left\|\boldsymbol{C}_{\boldsymbol{\delta}}^{1 / 2} \boldsymbol{w}\right\|}\right) \\
& \quad-\operatorname{erf}\left(\frac{\sqrt{1 / 2}-\operatorname{Re}\left\{\boldsymbol{w}^{H} \boldsymbol{a}\right\}}{\left\|\boldsymbol{C}_{\boldsymbol{\delta}}^{1 / 2} \boldsymbol{w}\right\|}\right) \geq 2(p-1) .
\end{aligned}
$$

The same steps can be applied to rewrite the second constraint of the problem (9) into its equivalent deterministic form.

To guarantee the robustness against errors in the sample estimate of the covariance matrix (3) that is also used in (9), it is meaningful to apply an additional non-adaptive diagonal loading with the factor $\gamma$, i.e., to use $\hat{\boldsymbol{R}}+\gamma \boldsymbol{I}$ instead of $\hat{\boldsymbol{R}}$, where $\boldsymbol{I}$ is identity matrix. The rationale for such additional non-adaptive diagonal loading could be explained as follows. According to (1), the data vectors $\boldsymbol{x}(k)$, $k=1, \ldots, K$ contain errors because of the mismatch of the steering vector $\boldsymbol{a}$. Hence, the mismatched sample covariance matrix can be written as

$$
\tilde{\boldsymbol{R}}=\frac{1}{K} \sum_{k=1}^{K}(\boldsymbol{x}(k)+\boldsymbol{e}(k))(\boldsymbol{x}(k)+\boldsymbol{e}(k))^{H}
$$

where $\boldsymbol{e}(k)$ is a random vector. Taking an expectation of $\tilde{\boldsymbol{R}}$ with respect to random vectors $\boldsymbol{e}(k)$ only, we obtain

$$
\begin{aligned}
\mathrm{E}\{\tilde{\boldsymbol{R}}\} & =\mathrm{E}\left\{\frac{1}{K} \sum_{k=1}^{K}(\boldsymbol{x}(k)+\boldsymbol{e}(k))(\boldsymbol{x}(k)+\boldsymbol{e}(k))^{H}\right\} \\
& =\hat{\boldsymbol{R}}+\frac{1}{K} \sum_{k=1}^{K} E\left\{\boldsymbol{e}(k) \boldsymbol{e}(k)^{H}\right\}=\hat{\boldsymbol{R}}+\gamma \boldsymbol{I}
\end{aligned}
$$




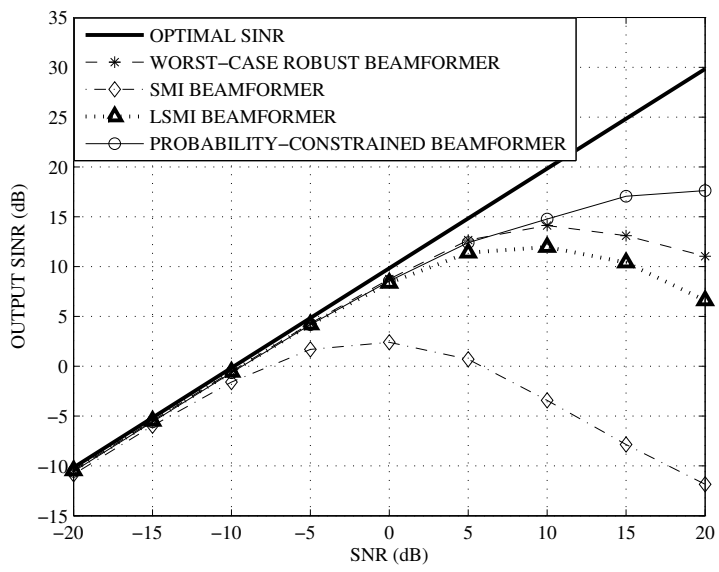

Fig. 1. Output SINR versus SNR. $K=100, \mathrm{INR}=40 \mathrm{~dB}$.

where $\boldsymbol{e}(k)$ is assumed to have zero mean and covariance $\gamma \boldsymbol{I}$. Note that (16) corresponds to the conventional fixed diagonal loading of $\hat{\boldsymbol{R}}$ with the loading factor $\gamma$.

Using (9) and taking into account (15) and (16), we obtain the following optimization problem

$$
\begin{aligned}
\min _{\boldsymbol{w}} \boldsymbol{w}^{H}(\hat{\boldsymbol{R}}+\gamma \boldsymbol{I}) \boldsymbol{w} \\
\text { subject to } \quad \operatorname{erf}\left(\frac{-\sqrt{1 / 2}-\operatorname{Re}\left\{\boldsymbol{w}^{H} \boldsymbol{a}\right\}}{\left\|\boldsymbol{C}_{\boldsymbol{\delta}}^{1 / 2} \boldsymbol{w}\right\|}\right) \\
-\operatorname{erf}\left(\frac{\sqrt{1 / 2}-\operatorname{Re}\left\{\boldsymbol{w}^{H} \boldsymbol{a}\right\}}{\left\|\boldsymbol{C}_{\boldsymbol{\delta}}^{1 / 2} \boldsymbol{w}\right\|}\right) \geq 2(p-1) \\
\quad \operatorname{erf}\left(\frac{-\sqrt{1 / 2}-\operatorname{Im}\left\{\boldsymbol{w}^{H} \boldsymbol{a}\right\}}{\left\|\boldsymbol{C}_{\boldsymbol{\delta}}^{1 / 2} \boldsymbol{w}\right\|}\right) \\
-\operatorname{erf}\left(\frac{\sqrt{1 / 2}-\operatorname{Im}\left\{\boldsymbol{w}^{H} \boldsymbol{a}\right\}}{\left\|\boldsymbol{C}_{\boldsymbol{\delta}}^{1 / 2} \boldsymbol{w}\right\|}\right) \geq 2(p-1) .
\end{aligned}
$$

The problem (17) is the so-called NLP problem. It can be efficiently solved using sequential quadratic programming (SQP) technique. The latter technique is an iterative approach in which each search direction is the solution of a particular quadratic programming (QP) subproblem [13]. The computational complexity of solving QP subproblem using, for example, the primal-dual potential reduction method is $\mathcal{O}\left(M^{4.5}\right)$ [14]. Note that the SQP algorithm has been implemented in MATLAB optimization toolbox.

\section{SIMULATIONS}

We assume a uniform linear array with $M=10$ omnidirectional sensors spaced half a wavelength apart, and two

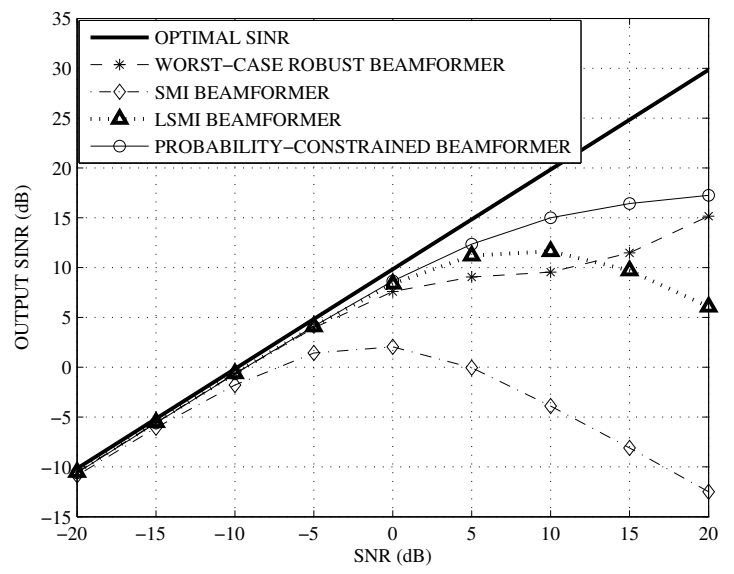

Fig. 2. Output SINR versus SNR. $K=100, \mathrm{INR}=20 \mathrm{~dB}$.

interfering sources with plane wavefronts and the directions of arrival $30^{\circ}$ and $50^{\circ}$, respectively. 100 Monte-Carlo runs are used to obtain each point in our simulations.

We consider the scenario with Ricean propagation medium where the mismatch vector $\boldsymbol{\delta}$ can be modelled as [1], [12]

$$
\boldsymbol{\delta}=\frac{\sigma_{\boldsymbol{\delta}}}{\sqrt{L}} \sum_{l=1}^{L} e^{j \psi_{l}} a\left(\theta_{0}+\theta_{l}\right)
$$

Here, $\sigma_{\delta}^{2}$ characterizes the total mismatch power, $L$ is the number of nonline-of-sight (NLOS) components due to scattering, $\psi_{l}$ is the phase shift parameter of $l$ th NLOS component, $\theta_{0}$ is the nominal direction-of-arrival (DOA) of a signal of interest, and $\theta_{l}$ is the angular shift of $l$ th NLOS component with respect to the nominal DOA. In our simulations, the parameters $\theta_{l}$ are independently drawn in each simulation run from a uniform random generator with the mean $\theta_{0}=3^{\circ}$ and standard deviation $\sigma_{\theta}=5^{\circ}$. The parameters $\psi_{l}$ are independently and uniformly drawn from $[0,2 \pi)$ in each run.

Four methods are compared: the proposed robust beamformer (17), the robust beamformer of [1], the SMI-MVDR beamformer of (5), and the Loaded SMI (LSMI) beamformer with fixed diagonal loading factor. As recommended in [1], $\varepsilon=3$ is chosen for the worst-case robust beamformer of [1] assuming that the nominal steering vector is normalized so that $\boldsymbol{a}^{H} \boldsymbol{a}=M$. For the LSMI and the proposed beamformers, the fixed diagonal loading parameter $\gamma=15$ is chosen. In the proposed beamformer, the parameter $p=0.95$ is taken. The covariance matrix $\boldsymbol{C}_{\boldsymbol{\delta}}$ is calculated as follows [12]

$$
\boldsymbol{C}_{\boldsymbol{\delta}}=\sigma_{\boldsymbol{\delta}}^{2} \int p(\theta) \boldsymbol{a}(\theta) \boldsymbol{a}^{H}(\theta) d \theta
$$




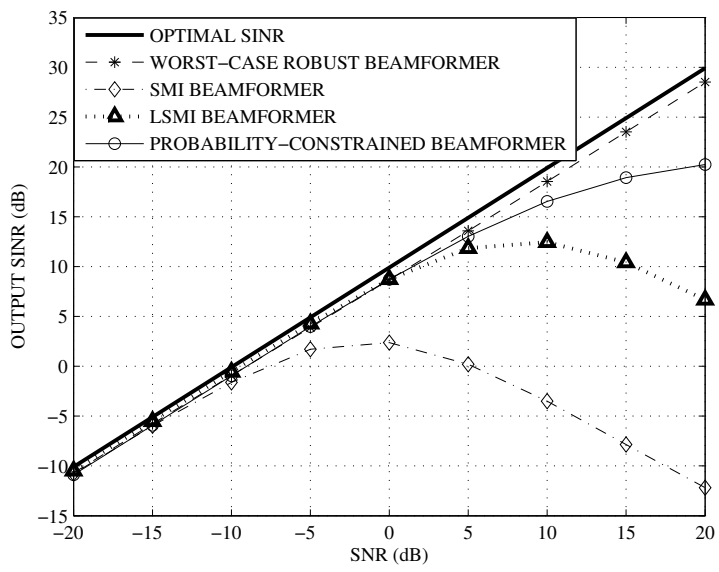

Fig. 3. Output SINR versus the SNR: $K=100, \mathrm{INR}=$ $5 \mathrm{~dB}$.

where $p(\theta)$ is the probability density function of $\theta$. Since $p(\theta)$ is assumed to be uniform, the $(k, l)$ th element of the covariance matrix $C_{\delta}$ can be calculated by numerical integration of the following integral

$\left[\boldsymbol{C}_{\boldsymbol{\delta}}\right]_{k, l}=\frac{\sigma_{\boldsymbol{\delta}}^{2}}{2 \sqrt{3} \sigma_{\theta}} \int_{\theta_{0}-\sqrt{3} \sigma_{\theta}}^{\theta_{0}+\sqrt{3} \sigma_{\theta}} \exp \left\{j 2 \pi \frac{d}{\lambda}(k-l) \sin \theta\right\} d \theta$

where $d$ is a distance between two neighboring sensors, and $\lambda$ is the wavelength. Moreover, $L=10$ is taken and the Ricean factor $\kappa=1 / \sigma_{\delta}^{2}=10$ is chosen, where the variance of the LOS components is normalized to one.

The output SINRs versus SNR for the methods tested are shown in the Figs. 1, 2 and 3 in the cases when the interference-to-noise ratio (INR) in a single sensor is equal to $40 \mathrm{~dB}, 20 \mathrm{~dB}$, and $5 \mathrm{~dB}$, respectively. In all figures, $K=$ 100. We can see that in the first two figures, the proposed beamformer has the best performance among all the techniques tested. These improvements are especially remarkable at high SNRs. However, the robust worst-case based adaptive beamformer of [1] performs better for INR $=5 \mathrm{~dB}$. Interestingly, the performance of the proposed beamformer does not depend significantly on the INR conditions, while the performance of the beamformer of [1] shows such a dependence.

Fig. 4 displays output SINR versus the sample size for $\mathrm{INR}=40 \mathrm{~dB}$ and $\mathrm{SNR}=15 \mathrm{~dB}$. We can see from this figure that the proposed beamformer shows the best performance for sufficiently large sample size.

In summary, the proposed probability-constrained optimization based beamformer performs better than the algorithm of [1] in most of our simulation examples. These performance improvements can be explained by the fact that

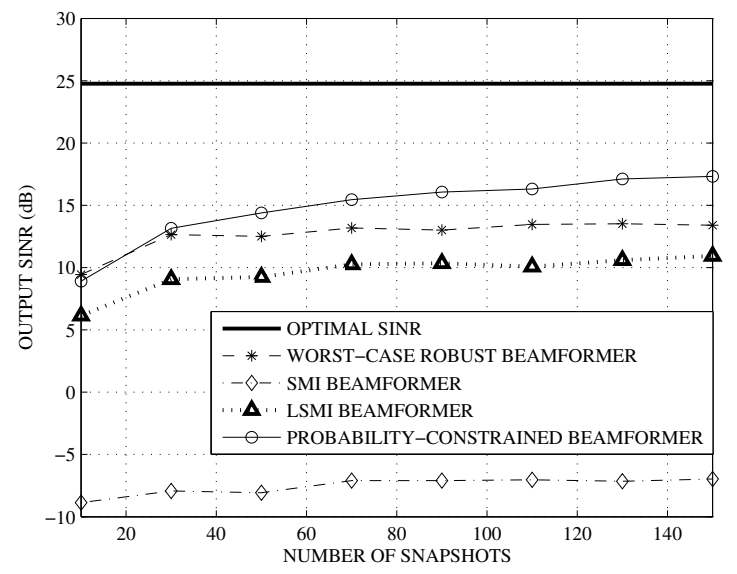

Fig. 4. Output SINR versus $K$. INR $=40 \mathrm{~dB}, \mathrm{SNR}=$ $15 \mathrm{~dB}$.

the proposed robust beamformer is less conservative than the worst-case approach of [1].

\section{CONCLUSIONS}

A new robust adaptive beamformer has been derived via probability-constrained optimization. To obtain the beamforming algorithm, we have converted the original probability-constrained optimization problem into an equivalent deterministic problem. To enable such a conversion, it has been assumed that the mismatch vector has Gaussian distribution. Based on this assumption, the original probabilityconstrained optimization problem has been reduced to the NLP problem that can be solved using SQP method. Simulation results have validated an excellent performance of the proposed technique as compared to several state-of-the-art robust beamforming algorithms.

\section{REFERENCES}

[1] S. A. Vorobyov, A. B. Gershman, and Z.-Q. Luo, "Robust adaptive beamforming using worst-case performance optimization: A solution to the signal mismatch problem," IEEE Trans. Signal Processing, vol. 51, pp. 313-324, Feb. 2003.

[2] R. G. Lorenz and S. P. Boyd, "Robust minimum variance beamforming," IEEE Trans. Signal Processing, vol. 53, pp. 1684-1696, May 2005.

[3] H. Cox, R. M. Zeskind, and M. H. Owen, "Robust adaptive beamforming," IEEE Trans. Acoust., Speech, and Signal Processing, vol. 35, pp. 1365-1376, Oct. 1987. 
[4] A. B. Gershman, "Robust adaptive beamforming in sensor arrays," AEU - Int. Journal Electron. Comm., vol. 53, pp. 305-314, Dec. 1999.

[5] Y. Rong, S. A. Vorobyov, and A. B. Gershman, "A robust linear receiver for multi-access space-time block coded MIMO systems based on probability-constrained optimization," Proc. VTC'04 - Spring, Milan, Italy, May 2004, vol. 1, pp. 118-122.

[6] Y. Rong, S. A. Vorobyov, and A. B. Gershman, "Robust linear receiver design for multi-access space-time block coded MIMO systems using stochastic optimization," Proc. IEEE Workshop on Statistical Signal Processing, Bordeaux, France, July 2005.

[7] A. Prékopa, Stochastic Programming. Dordrecht, Netherlands: Kluwer Academic Publishers, 1995.

[8] R. A. Monzingo and T. W. Miller, Introduction to Adaptive Arrays. New York: Wiley, 1980.

[9] J. Li, P. Stoica, and Z. Wang, "On robust Capon beamforming and diagonal loading," IEEE Trans. Signal Processing, vol. 51, pp. 1702-1715, July 2003.
[10] O. Besson and F. Vincent, "Performance analysis of beamformers using generalized loading of the covariance matrix in the presence of random steering vector errors," IEEE Trans. Signal Processing, vol. 53, pp. 452-459, Feb. 2005.

[11] A. B. Gershman, C. F. Mecklenbräuker, and J. F. Böhme, "Matrix fitting apprach to direction of arrival estimation with imperfect coherence of wavefronts," IEEE Trans. Signal Processing, vol. 45, pp. 1894-1899, July 1997.

[12] G. Fuks, J. Goldberg, and H. Messer, "Bearing estimation in a Ricean channel. Part I: Inherent accuracy limitations," IEEE Trans. Signal Processing, vol. 49, pp. 925-937, May 2001.

[13] D. P. Bertsekas, Nonlinear Programming. Belmont, Athena Scientific, 1995.

[14] Y. Nesterov and A. Nemirovski, Interior Point Polynomial Algorithms in Convex Programming. Philadelphia, PA: SIAM, 1994. 